\title{
Quantitative assessment of radiation- induced changes of bladder and rectum collagen structure using optical methods
}

Marina Kochueva

Varvara Dudenkova

Sergey Kuznetsov

Angelina Varlamova

Ekaterina Sergeeva

Elena Kiseleva

Anna Maslennikova 


\title{
Quantitative assessment of radiation-induced changes of bladder and rectum collagen structure using optical methods
}

\author{
Marina Kochueva, ${ }^{\mathrm{a}, *}$ Varvara Dudenkova, ${ }^{\mathrm{b}}$ Sergey Kuznetsov, ${ }^{\mathrm{c}}$ Angelina Varlamova, ${ }^{\mathrm{d}}$ Ekaterina Sergeeva, ${ }^{\mathrm{e}}$ \\ Elena Kiseleva, ${ }^{b}$ and Anna Maslennikova, ${ }^{a, d}$ \\ aNizhny Novgorod State Medical Academy (NNSMA), Department of Oncology, Radiation Therapy, Radiation Diagnostics, \\ Nizhny Novgorod, Russia \\ bNNSMA, Institute of Biomedical Technologies, Laboratory of Studying Optical Structure of Biotissues, Nizhny Novgorod, Russia \\ 'NNSMA, Department of Pathological Anatomy, Nizhny Novgorod, Russia \\ 'Lobachevsky State University, Institute of Biology and Biomedicine, Department of Biophysics, Gagarin Avenue Nizhny Novgorod, Russia \\ enstitute of Applied Physics RAS, Laboratory for Optical Techniques, Department for Radiophysics Methods in Hydrophysics, Novgorod, Russia
}

\begin{abstract}
The objective of the study is the quantitative analysis of the dose-time dependences of changes occurring in collagen of bladder and rectum after gamma-irradiation using optical methods [nonlinear microscopy in a second harmonic generation (SHG) detection regime and cross-polarization optical coherence tomography (CP OCT)]. For quantitative assessment of the collagen structure, regions of interest on the SHG-images of two-dimensional (2-D) distribution of SHG signal intensity of collagen were chosen in the submucosa. The mean SHG signal intensity and its standard deviation were calculated by ImageJ 1.39p (NIH). For quantitative analysis of CP OCT data, an integral depolarization factor (IDF) was calculated. Quantitative calculation of the SHG signal intensity and the IDF can provide additional information about the processes of the collagen radiation-induced degradation and subsequent remodeling. High positive correlation between the mean SHG signal intensity and the mean IDF of bladder and rectum demonstrates that CP OCT can be used as an "optical biopsy" in the grading of collagen radiation damage. ๑ 2018 Society of Photo-Optical Instrumentation Engineers (SPIE) [DOI: 10.1117/1.JBO.23 .9.091417]
\end{abstract}

Keywords: collagen; radiation-induced adverse events; nonlinear microscopy; second harmonic generation; cross-polarization optical coherence tomography.

Paper 180032SSRR received Jan. 16, 2018; accepted for publication Jul. 20, 2018; published online Aug. 22, 2018.

\section{Introduction}

Despite the development of the methods of conformal irradiation of malignant tumors, adverse events in normal tissues that arise in the early and late periods after radiation therapy are still a challenge of radiation oncology. ${ }^{1}$ The severity of the side effects of radiation/chemoradiation therapy can range from minor functional disorders to severe injuries that have a significant impact on the patients' quality of life and lead to disability. ${ }^{2}$

One of the key mechanisms for the development of normal tissues' radiation damage is degradation and subsequent remodeling of the connective-tissue matrix. The collagen synthesis activation in irradiated tissues leads to the radiation-induced fibrosis, which is typical for second grade of radiation changes and accompanied by the corresponding organs dysfunction. ${ }^{3}$ The main manifestation of the third and fourth grades of complications is the degradation of extracellular matrix components, which leads to the subsequent appearance of necrosis, bleeding, and fistula. ${ }^{4,5}$ All modern systems of grading the severity of radiation-induced organ and tissue damage are based on patient complaints, symptoms, and visual changes; these cannot provide objective and quantifiable information. ${ }^{1}$ From this point of view, a noninvasive method for detecting quantifiable changes in the connective-tissue matrix of organs included

*Address all correspondence to: Marina Kochueva, E-mail: marina.kochueva@ mail.ru into the irradiated volume during the radiation therapy of malignant tumors is highly desirable.

The development of optical methods [optical coherence tomography (OCT), ${ }^{6,7}$ nonlinear microscopy $\left.{ }^{8,9}\right]$ has opened new opportunities for studying and assessing the structure of the extracellular matrix ex vivo and in vivo. Visualization of collagen using the method of nonlinear microscopy occurs due to the phenomenon of the second harmonic generation (SHG) from molecules that do not have a center of symmetry. ${ }^{10-12}$ Under the short-pulse $(\sim 100 \mathrm{fs})$ laser radiation with a high peak power, collagen generates a nonlinear optical response at a doubled frequency. ${ }^{13,14}$ Scanning with a fine-focused laser beam makes it possible to obtain images that reflect the peculiarities of the collagen folding in the tissue in detail. Nonlinear microscopy in SHG detection regime is now used in dermatology, ${ }^{15}$ ophthalmology, ${ }^{16}$ oncology,,${ }^{17,18}$ atherosclerotic vascular disease studies, ${ }^{19,20}$ and diseases with abnormal accumulation of collagen in tissues and organs. ${ }^{21}$

The principle of OCT is the measurement of the echo time delay and magnitude of backscattered light. The OCT operation is based on light interferometry. ${ }^{22}$ The method is widely used in a routine clinical practice for diagnostics and monitoring. ${ }^{23}$ One of its modifications is the cross-polarization OCT (CP OCT), which can obtain specific information about the structure of extracellular matrix based on the polarization properties of

$1083-3668 / 2018 / \$ 25.00$ (c) 2018 SPIE 
the tissue. ${ }^{24}$ Optical methods have been already used in assessing the features of tissues exposed to ionizing radiation. The previous studies using the CP OCT have shown that early radiation complications of bladder are located primarily in the zone of connective-tissue structures under the urothelium. ${ }^{25}$ The study of the radiation-induced changes of the collagen on the model (rat's tail tendon) using optical and physical-chemical methods revealed the main mechanisms of its degradation and subsequent remodeling depending on the dose and the time after irradiation. ${ }^{26}$ The combination of nonlinear microscopy and OCT can be used to diagnose various pathological processes in real time, avoiding unnecessary biopsies. ${ }^{27-30}$

CP OCT imaging is a minimally invasive procedure that has been performed using a sterile endoscopic OCT-probe under a visual control of a cystoscope with a very low risk of complications. ${ }^{31}$ However, to use this technique as the method for the evaluation of tissues damage degree after the irradiation, the verification of the CP OCT results with histology is needed. Nonlinear microscopy allows to obtain objective information about the collagen structure, i.e., the rate of change that characterizes orientation, the degree of structural organization of collagen on fiber and fibrils levels, and it allows to judge about the presence of pathology. ${ }^{32}$ The correlation between these two methods will further help to determine the nature of collagen changes caused by radiation therapy using the "optical biopsy" itself, without performing a histological study.

The study objective was the quantitative analysis of the dosetime dependences of changes occurring in collagen of bladder and rectum after gamma-irradiation using two optical methods: nonlinear microscopy (in the regime of SHG detection) and cross-polarization OCT.

\section{Materials and Methods}

\subsection{Study Object}

Experiments were performed on white outbred rats (a total of 20 animals) kept in the standard vivarium conditions. The studies were performed in accordance with the ethical principles established by the European Convention for the Protection of Vertebrate Animals used for experimental and other scientific purposes with the permission of the Local Ethical Committee of the Nizhny Novgorod State Medical Academy (Protocol no. 9, October 15, 2014). Before procedure, intraperitoneal anesthesia was performed with a Zoletil solution in a dose of $50 \mathrm{mg} / \mathrm{kg}$, then the animals were fixed on a special plate. Irradiation was carried out in vivo using the $\mathrm{Co}^{60}$ unit "Terabalt" (UJP, Prague, Czech Republic) with a mean beam energy of $1.25 \mathrm{MeV}$, by a local field, by single doses equivalent to 2 , 10 , and $40 \mathrm{~Gy}$, respectively. The exposure time was calculated in accordance with the size of the irradiation field, the depth, and the single dose. In $24 \mathrm{~h}, 1$ week, and 1 month after exposure, the animals were withdrawn from the experiment using ether anesthesia.

Bladder and rectum were dissected and ex-vivo CP OCT study was performed immediately on the entire surface of the organs. The samples were constantly wetted with $0.9 \% \mathrm{NaCl}$ solution to avoid drying. The preparations of the bladder and rectum of two unirradiated animals were used as controls. A part of the organ was separated for microscopic examination before the CP OCT procedure. The samples for the nonlinear microscopy and light microscopy were fixed for $24 \mathrm{~h}$ in a $10 \%$ buffered solution of neutral formalin. Then, the samples were embedded into paraffin ("Histomix-extra," "BioVitrum," Russia), followed by the mounting of paraffin blocks. The slices of 0.3 - and $10-\mu \mathrm{m}$-thick were produced from the resulting blocks using the Leica 450 RM rotary microtome (Leica Microsystems, Germany). The slices of $0.3-\mu \mathrm{m}$-thick were stained with picrofuchsin according to van Gieson and were used to verify the results obtained by the nonlinear microscopy. The preparations were studied with a Leica DMLS microscope (Leica Microsystems, Germany). The samples of $10-\mu \mathrm{m}$-thick obtained from the same blocks were dewaxed and investigated using the method of the nonlinear microscopy without additional staining.

\subsection{Nonlinear Microscopy: Imaging and Processing}

Studies of unstained slices of bladder and rectum samples were performed on an inverted laser scanning microscope LSM Axiovert 510 Meta (Carl Zeiss, Germany). A short-pulse femtosecond laser MaiTai HP (Spectra Physics) with a pulse repetition rate of $80 \mathrm{MHz}$ and a duration of about 100 femtoseconds was used. Deparaffined slices placed on a slide were covered with a cover glass of $170-\mu \mathrm{m}$ thick. The images of the structure were constructed with the oil-immersion lens EC Plan-Neofluar with 40-fold magnification and a numerical aperture of 1.3, which made it possible to obtain a field of $318 \times 318$ microns with a resolution of $1024 \times 1024$ pixels. The following spectral characteristics of the visualization system were used: excitation at a wavelength of $800 \mathrm{~nm}$, detection in the wavelength range of 362 to $415 \mathrm{~nm}$, containing a peak of the nonlinear response of collagen at the second harmonic $(400 \mathrm{~nm}) .{ }^{13}$ Registration was carried out with the fully open pinhole.

To quantify the features of the collagen fibers, the regions of interest (ROI) on the submucosal layer were selected (Fig. 1). The areas corresponding to the quality criteria (the absence of blood vessels, epithelium, and muscle fibers in the area of interest and the absence of dark fields corresponding to areas that were out of focus) were outlined. In total, 109 images of the bladder and 145 images of the rectum were obtained. In total, 265 ROI on the bladder SHG-images and 343 ROI on the rectum SHG-images were analyzed.

The parameter of the SHG signal intensity, which represents the average SHG signal intensity on all informative pixels in the image for the selected ROI, has been used as a quantitative criterion for assessing the structure of collagen depending on the dose and time after radiation exposure. The average intensity of the intact bladder/intact rectum SHG signal was obtained as a result of averaging the intensity values from a set of ROI. All the values obtained were normalized to the average value of the intensity of the SHG signal of intact samples taken as unity. The average value of the SHG signal intensity from all the analyzed ROI was calculated for each dose and time after irradiation, the result being presented as mean \pm SD. Calculations were made in the ImageJ $1.39 \mathrm{p}$ software (NIH, USA). All tissue samples were fixed uniformly in a neutral formalin; the values of the signal intensity distribution of every sample were normalized to the average value of the intensity of the SHG signal of intact samples taken as unity. There are data that formalin fixation could affect a collagen structure. ${ }^{33}$ Although the protocol of tissue processing was the same for all samples, we suggest that the resulting changes did not influence on the relative values of the signal intensity distribution on the nonlinear microscopy images. All data were obtained from the slices of the same 


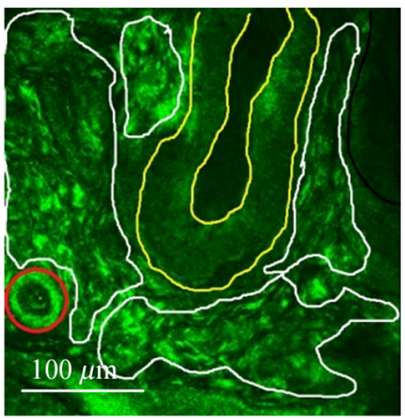

(a)

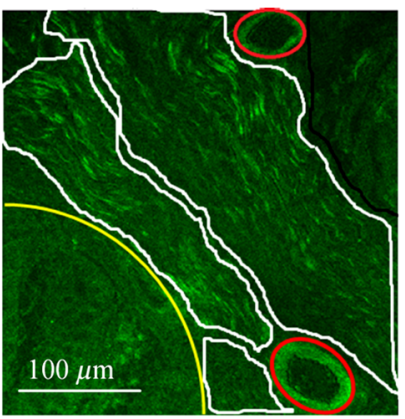

(b)

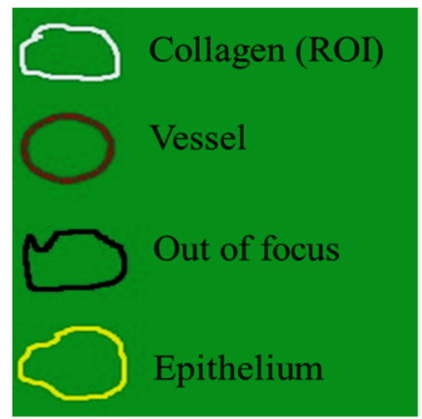

(c)

Fig. 1 Examples of ROI selection. (a) Intact bladder SHG-image, (b) intact rectum SHG-image, and (c) contours.

thickness at the same parameters of the pump power, digital amplification, and the dynamic range of the recorded signal.

\subsection{Cross-Polarization Optical Coherence Tomography: Imaging and Processing}

The cross-polarization OCT was used to assess the changes in the structure of biological tissues at the level of general organs (bladder and rectum) architectonics. The device "OCT 1300-U" (IAP RAS, Nizhny Novgorod, Russia) was used. ${ }^{34}$

The wavelength of the superluminescent diode used as the radiation source was $1315 \mathrm{~nm}$, the spectral width was $60 \mathrm{~nm}$, which determined the spatial resolution at a depth of $20 \mu \mathrm{m}$. The tomograph is equipped with a flexible fiber-optic probe with an end-window of an optical scanner of $2.7 \mathrm{~mm}$ in diameter, with a lateral resolution of $25 \mu \mathrm{m}$. When scanning the ROI, the probe was brought into a contact with the object of investigation. Before OCT-scanning, the dissected organs were spread and fixed on the plate. OCT imaging was performed "step by step" all over the organ surface. From every organ, at least 15 images were obtained. The averaged data from all obtained images for every time and dose point have been presented in the paper.

The CP OCT system has a common-path optical layout and two signal acquisition channels: one for scattered light that maintained initial polarization ("copolarized"), and one that now exhibits orthogonal polarization ("cross polarized"). ${ }^{23}$ The size of the CP OCT image in each polarization is 200 points in the transverse direction and 256 points in depth, which corresponds to $2.2 \times 1.3 \mathrm{~mm}^{2}$. The brightest areas of the CP OCT image represented in the brown palette correspond to the maximum intensity signal, and the darkest ones correspond to the signal with minimum intensity. ${ }^{34}$

A dimensionless parameter, the integral depolarization factor (IDF), which is the ratio of the received OCT signal powers, averaged over all informative pixels of the CP OCT image, in the orthogonal and original channels. ${ }^{31}$ The amplitude of the OCT signal in the orthogonal polarization depends on the degree of inverse cross scattering of the probe radiation by the tissues that are studied. ${ }^{35}$ The main structural cross-scattering components are collagen fibers. The degree of cross-scattering depends on their structural and spatial organizations. Therefore, the IDF magnitude can be used to judge the structure of collagen fibers in the tissue, and, consequently, to assess the degree of radiation-induced damage of the organs' extracellular matrix.

\subsection{Statistical Analysis}

Statistical analysis was performed by ImageJ $1.39 \mathrm{p}(\mathrm{NIH})$ and Microsoft Excel 2007 software. The intact samples and the samples obtained at various times after irradiation (a day, a week, and a month) by the doses of 2, 10, and 40 Gy were compared using the one-tailed student's t-test in Statistica 6.0 software. $P$ values $<0.05$ were considered to be statistically significant. Correlation analysis was performed between the mean values of the intensity of the SHG signal determined by a nonlinear microscopy method and the values of IDF were determined by CP OCT for bladder and rectum separately. A correlation cloud was created and linearly approximated by the Prism software (GraphPad Software Inc., La Jolla). The Spearman's rank correlation coefficient and the significance level were calculated.

\section{Results}

\subsection{Results of the Study of Radiation-Induced Changes in Bladder Collagen}

On the images of intact rat bladder obtained using the nonlinear microscopy in an SHG detection regime, the main localization of collagen fibers were the urothelium basal membrane, the submucosa, the walls of the blood vessels, and the framework of the muscle layer [Fig. 1(a)]. ${ }^{36}$

No changes in the extracellular matrix were detected during the visual assessment of images of the SHG signal distribution of collagen of the bladder wall after exposure to a dose of 2 Gy irrespective of the time after irradiation (Fig. 2). The main manifestations of radiation damage to collagen on the images after irradiation at doses of 10 and 40 Gy were swelling of the submucosal layer, fragmentation of collagen fibers, destruction of connective-tissue structures, and more pronounced after exposure to a dose of 40 Gy (Fig. 2). ${ }^{36}$

A clear dependence of the SHG signal intensity of the bladder wall collagen on the dose and the time after irradiation was observed after the quantitative assessment of SHG-images. After irradiation at a dose of $2 \mathrm{~Gy}$, the values of the intensity of the SHG signal did not differ from the intact bladder samples irrespective of the term after irradiation. One day after irradiation, the decrease in the intensity of the SHG signal of collagen after irradiation in doses of 10 and $40 \mathrm{~Gy}$, respectively, was observed (Fig. 3). Statistically significant difference $(p=0.05)$ compared with the intact sample was revealed after irradiation at a dose of 40 Gy. 
1 day
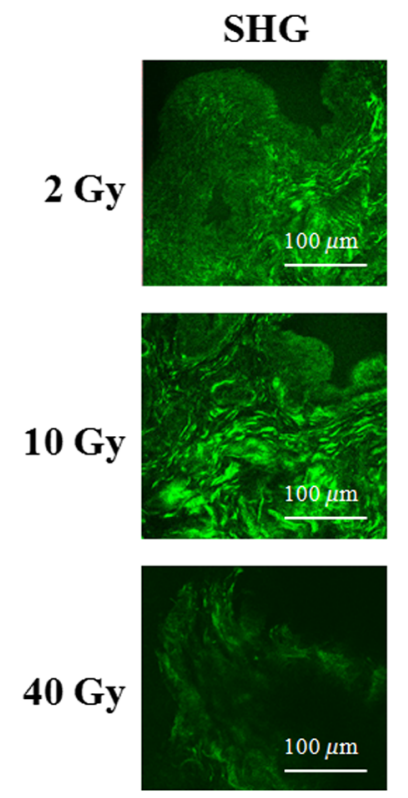

1 week
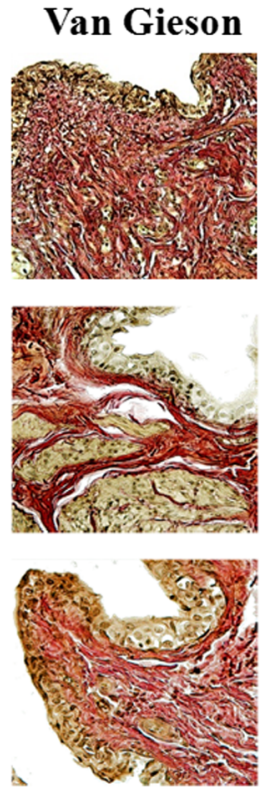

SHG
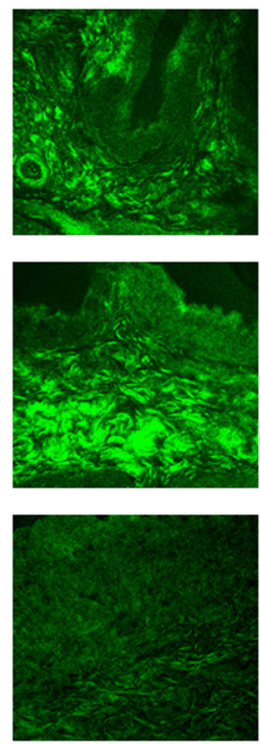

Van Gieson
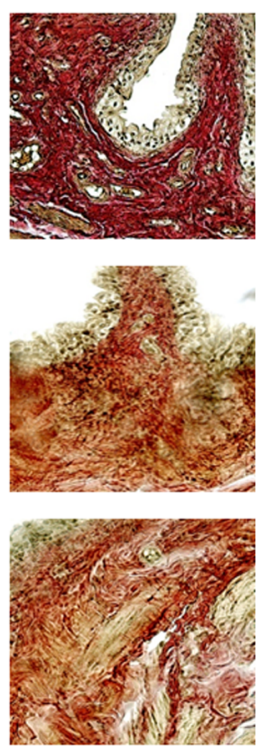

1 month
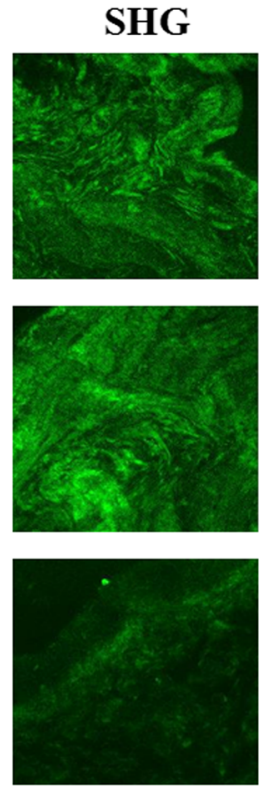

Van Gieson
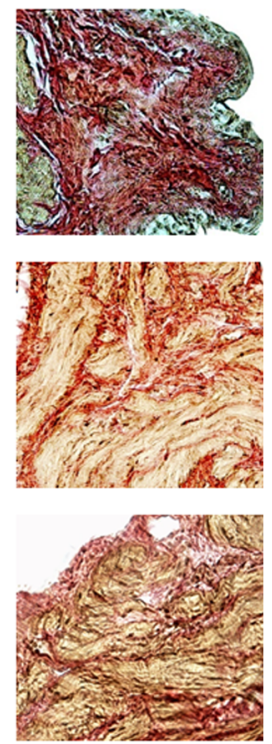

Fig. 2 Images of the bladder depending on the dose and time after irradiation. Left columns-SHGimages (nonlinear microscopy in SHG detection regime, image size $318 \times 318 \mu \mathrm{m}$, oil-immersion); right columns—van Gieson staining (40× objective magnification).

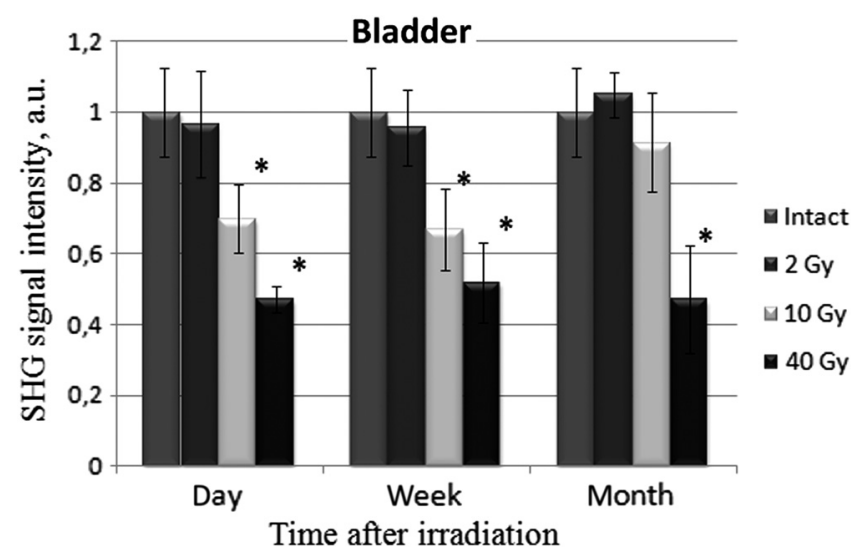

Fig. 3 Dynamics of the SHG signal of collagen depending on the dose and time after radiation exposure. Normalized mean intensity of the SHG signal in ROI of the bladder. "Statistically significant difference from the initial level.
A week after the exposure, a statistically significant decrease in the intensity of the SHG signal to $0.67 \pm 0.12$ a.u. after irradiation at a dose of $10 \mathrm{~Gy}$ and to $0.52 \pm 0.11$ a.u. after 40 Gy irradiation remained in comparison with the intact sample (1 \pm 0.13 a.u.) (Fig. 3). A month after irradiation at a dose of $10 \mathrm{~Gy}$, the intensity of the SHG signal was almost restored to the level of the intact sample $-0.91 \pm 0.14$ a.u., whereas the signal intensity of the samples irradiated at a dose of $40 \mathrm{~Gy}$ did not even show a tendency to return to the initial level and amounted to $0.47 \pm 0.15$ a.u. (Fig. 3).

The four layers can be differentiated on OCT images of intact bladder samples of rats in the initial polarization, corresponding to the layers on histological image: the upper layer with a moderate signal level corresponding to the urothelium; medium layer with a high signal level corresponding to the connective-tissue layer (proper mucous plate and submucosal layers); the next layer with a moderately intense signal corresponding to the muscle layer; and the lowest layer corresponding to the outer adventitious membrane [Figs. 4(a) and 4(c)]. In the orthogonal polarization, in these zones of the bladder, a distinct signal is

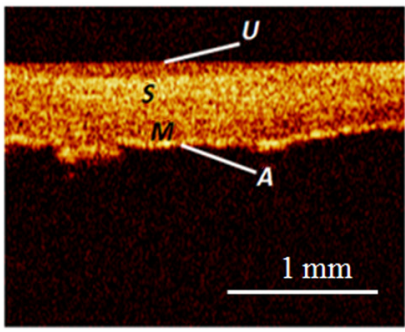

(a)

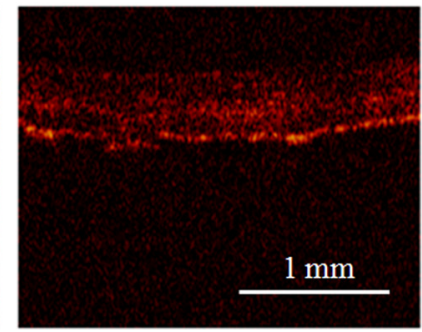

(b)

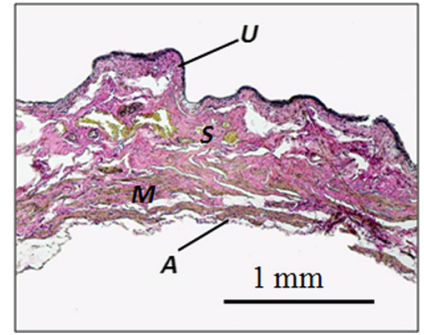

(c)

Fig. 4 OCT-image of intact bladder: (a) initial polarization, (b) orthogonal polarization, (c) standard histological image of bladder, HE staining. ( $U$, urothelium; $S$, submucosa, and lamina propria; $M$, muscle; and $A$, adventitia) 
clearly determined from collagen fibers in the proper mucous plate, the submucosa layer, and the adventitious membrane. The urothelium does not have a visible OCT signal as the transitional epithelium does not change the polarization of the probing radiation [Fig. 4(b)].

There have been no visible changes in the structure identified after any dose expose, but the numerical image processing revealed changes (see Figs. 5 and 7). The IDF value of the intact bladder was $0.067 \pm 0.01$ a.u. After irradiation at a dose of $2 \mathrm{~Gy}$, the changes in the IDF were not statistically significant irrespective of the period after irradiation (Fig. 5). A day after irradiation of the bladder in doses of 10 and 40 Gy, respectively, the IDF values reduced to $0.053 \pm 0.01$ and $0.05 \pm 0.01$, respectively. A week after irradiation in doses of 10 and 40 Gy, the IDF values remained lower, which indicated the continuing processes of the connective-tissue matrix degradation. An increase in the values

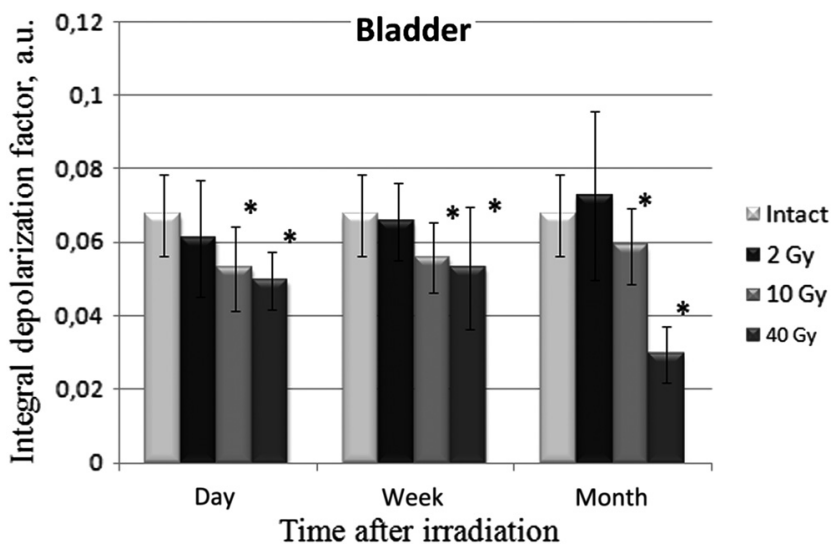

Fig. 5 Mean IDF depending on the dose and time after radiation exposure. "Statistically significant difference from the initial level. of the IDF almost to the initial level was observed 1 month after the exposure at a dose of $10 \mathrm{~Gy}$. There was a pronounced decrease in the IDF to $0.029 \pm 0.011$ month after the radiation exposure at a dose of 40 Gy (Fig. 5). Statistically significant differences compared with the unirradiated control were detected in a day, a week, and a month after a radiation exposure in the doses of 10 and $40 \mathrm{~Gy}$, respectively.

The analysis of the data showed a high positive correlation (the Pearson correlation coefficient was 0.855 at a significance level of $p<0.005$ ) between the values of the SHG signal intensity and the IDF values of the bladder wall. The linear approximation showed a satisfactory value of the approximation reliability $\left(R^{2}>0.7\right)$. This indicates that CP OCT with the bladder tissue IDF calculation can be used as a method for verifying the dynamics of changes in collagen structures after the ionizing radiation exposure.

\subsection{Results of the Study of Radiation-Induced Changes in Rectal Collagen}

The basal membrane, a proper mucous plate, submucosa and walls of blood vessels, and the stromal skeleton muscle sheath are the main allocations of connective-tissue structures in intact rat rectum [Fig. 1(b)].

Visual changes in the rectum wall collagen arising from 1 day up to 1 month after exposure in the studied doses were similar to the changes in the bladder wall collagen structures (Fig. 6).

Quantitative analysis of the SHG images of rectum samples showed a decrease in the intensity of the collagen SHG signal to $0.86 \pm 0.18$ a.u. a day after irradiation at a dose of $10 \mathrm{~Gy}$ and to $0.74 \pm 0.04$ a.u. after $40-G y$ irradiation (Fig. 7). The maximum decrease in the signal intensity was observed after exposure to a dose of $40 \mathrm{~Gy}$ (difference in comparison with the intact sample was statistically significant, $p=0.05$ ). A week after the
1 day

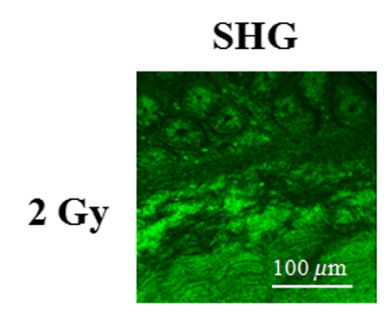

10 Gy

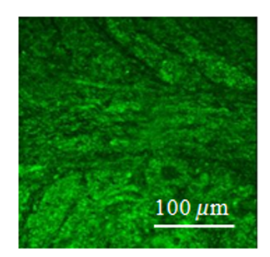

40 Gy

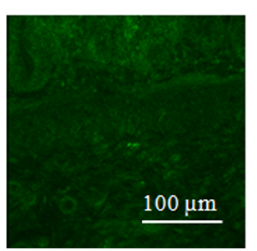

1 week
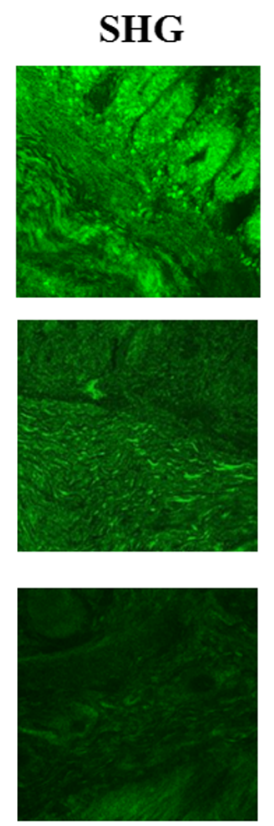
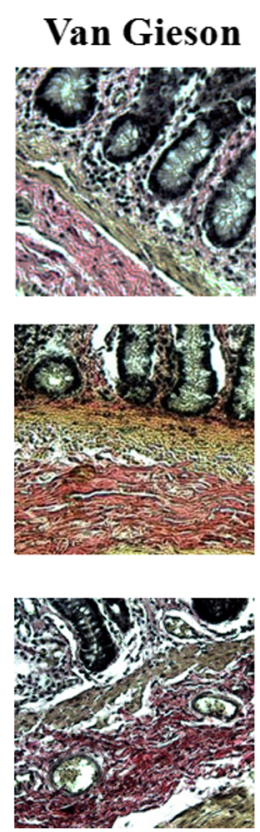

\section{1 month}
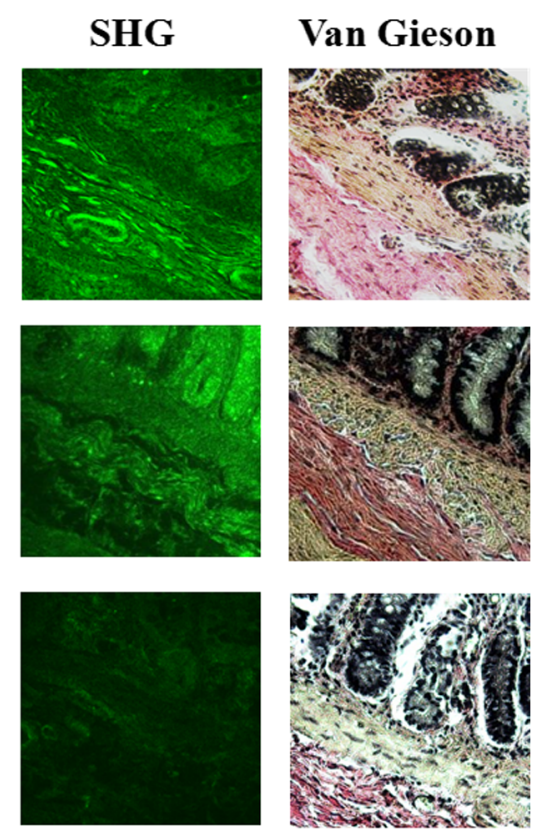

Fig. 6 Images of the rectum depending on the dose and time after irradiation. Left columns-SHGimages (nonlinear microscopy in SHG detection regime, image size $318 \times 318 \mu \mathrm{m}$, oil-immersion); right columns-van Gieson staining (40x objective magnification). 


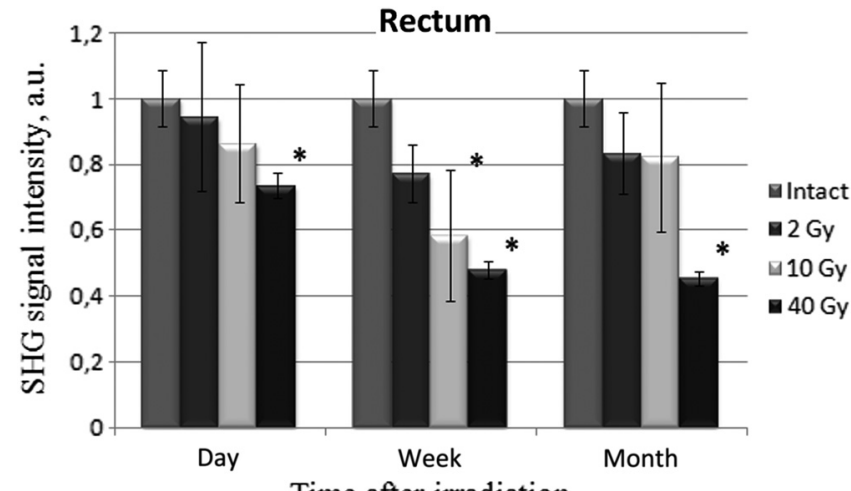

Time after irradiation

Fig. 7 Dynamics of SHG signal of collagen depending on the dose and time after radiation exposure. Normalized mean intensity of SHG signal in ROI of the rectum. "Statistically significant difference from the initial level.

exposure, the decrease in the SHG signal remained, having statistically significant differences compared with unirradiated control after exposure to a dose of $10 \mathrm{~Gy}(0.58 \pm 0.2$ a.u. $)$ and 40 (0.48 \pm 0.03 a.u.), respectively. A tendency of the SHG signal intensity returning to the original level was observed a month after exposure to a dose of $10 \mathrm{~Gy}$, and signal intensity of the samples irradiated in a dose of 40 Gy remained significantly reduced: $0.45 \pm 0.02$ a.u. Irradiation in a dose of $2 \mathrm{~Gy}$ did not significantly affect the SHG signal intensity irrespective of the period after exposure.

Four layers are differentiated on OCT images of intact rectum samples in the initial polarization, corresponding to the layers on a histological image: the upper layer with a moderate signal level corresponding to the epithelium; the second with a high signal level corresponding to the submucosal layer; the third one with a moderate signal level - a muscular layer; the lower thin layer with an uneven border-a serous layer [Figs. 8(a) and $8(\mathrm{c})$ ]. In the orthogonal polarization, in these zones of the rectum, a distinct signal is clearly determined from collagen fibers in the submucosa layer and the serous membrane. In this case, the epithelium does not demonstrate a visible signal [Fig. 8(b)].

No visible changes in the structure were revealed on CP OCT images of rectum samples obtained at various times after irradiation in the studied dose range. After the quantitative analysis of CP OCT images of the intact rectum, the mean IDF value was $0.058 \pm 0.01$. A day after irradiation of the rectum in doses of 2,10 , and $40 \mathrm{~Gy}$, the IDF values reduced to $0.051 \pm 0.01$, $0.044 \pm 0.01$, and $0.046 \pm 0.01$, respectively. In a week and a month after irradiation in doses of 2 and 10 Gy, the IDF values slightly increased. After a dose expose of $40 \mathrm{~Gy}$, the IDF values

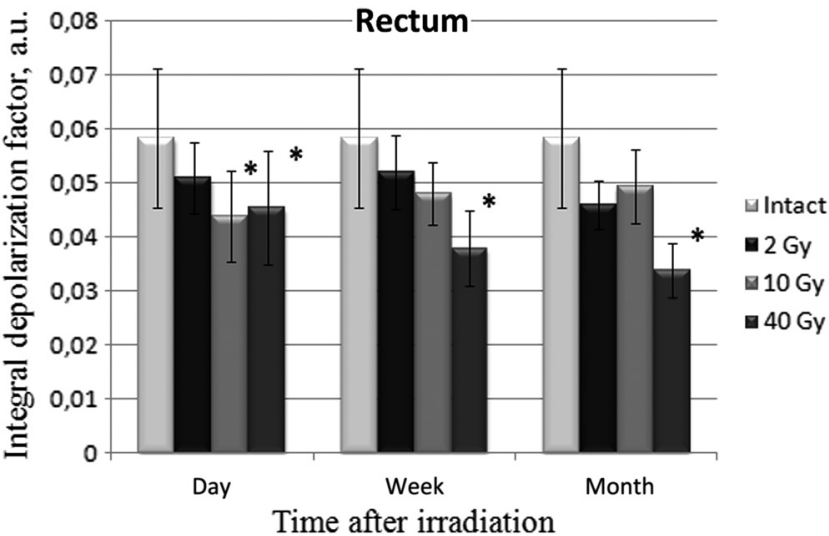

Fig. 9 Mean IDF depending on the dose and time after radiation exposure. "Statistically significant difference from the initial level.

decreased progressively and amounted to $0.38 \pm 0.01$ in a week and $0.034 \pm 0.01$ in a month, which indicated the persistent connective-tissue matrix degradation processes (Fig. 9). There were statistically significant changes compared with intact control in a day after 10-Gy exposure, and in a day, a week, and a month after irradiation in the dose of 40 Gy.

The analysis of the data showed a high positive correlation between the values of the SHG signal intensity and the IDF values (the value of the linear Pearson correlation coefficient was $p=0.813$, with a significance level of $p<0.005$ ). The linear approximation showed a satisfactory value of the approximation reliability $\left(R^{2}>0.6\right)$.

\section{Discussion}

The study objective was to quantify the features of the connective-tissue matrix of internal organs (bladder and rectum) at different times after exposure to increasing clinically relevant doses of ionizing radiation. Nonlinear microscopy images with the SHG signal distribution were subjected to a quantitative analysis with an assessment of the SHG signal intensity from collagen, depending on the dose and the time after irradiation. A choice of a correct approach for an objective assessment of collagen structure was one of the primary objectives of the work. At present, there are several different approaches, most of them are based on the assessment of characteristic features of the fibers, such as a size, shape, arrangement, and proportions of its parts. The key features on which the methods of quantitative analysis are based, are the number of fibers, their organization and disorganization, the density of packing, the curvature of the fibers, their longitudinal and transverse dimensions. ${ }^{37}$ To quantify the density and

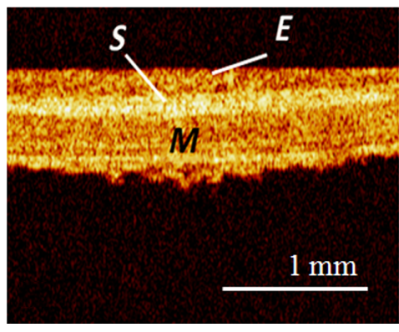

(a)

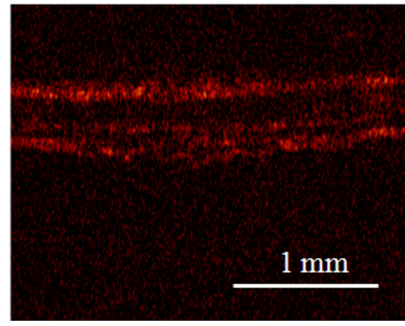

(b)

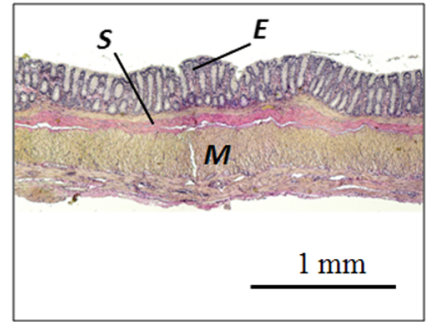

(c)

Fig. 8 OCT-image of intact rectum: (a) initial polarization, (b) orthogonal polarization, (c) standard histological image of rectum, HE staining. ( $E$, epithelium; $S$, submucosa; and $M$, muscle) 
orientation of collagen structure, the most used method is the fast Fourier transform. ${ }^{38}$

In addition, a combination of the Fourier transforms and the Hough transform ${ }^{39}$ and other modifications of the method ${ }^{40}$ can be used. For cases with a poorly expressed order of collagen fibers, a gray-level co-occurrence matrix is also used. ${ }^{41}$ For quantitative analysis of tissues that do not have certain features of collagen structure, the most often used parameter is the average signal intensity and its standard deviation. It can be considered as the most universal and objective as the intensity of the signal is calculated directly from the initial data and does not require subjective variation in any parameters. This very approach was used in our work to objectify the data obtained by visual assessment of the nonlinear microscopy images.

Both histological study and nonlinear microscopy in SHG detection regime revealed two parallel processes in the bladder and rectum: destruction (disorganization) and restoration of damaged structures of collagen-containing elements. Disorganization of the extracellular matrix observed with van Gieson microscopy manifested itself as a diffuse signal decrease in the range of 362 to $415 \mathrm{~nm}$ on images of SHG signal distribution. Similar changes (decrease in the intensity of the collagen SHG signal) were detected in vivo when monitoring the skin after a thermal burn, ${ }^{8}$ as well as in systemic diseases characterized by disorganization of connective-tissue structures of internal organs. ${ }^{31}$ A distinct dependence of changes in the collagen SHG signal on the radiation dose was revealed. A dose of 2 Gy did not cause significant changes in the signal intensity, whereas exposure in doses of 10 and 40 Gy led to a statistically significant reduction of it in a day and a week after exposure.

The recovery mechanisms are triggered as a response to the destruction of connective-tissue structures: the neocollagenesis process, the start of which was noted in a month after irradiation of a dose of $10 \mathrm{~Gy}$. These processes were reflected on the images of the SHG signal distribution in the form of signal intensity growth after its decrease. The remodeling of the extracellular matrix is significantly slowed after exposure to higher doses (40 Gy).

Today the development of nonlinear microscopy technology goes in the direction of in-vivo diagnosis of chronic inflammatory processes, precancerous diseases, and malignant tumors of the colon, esophagus, stomach, and skin diseases. However, there can be some problems in this area, the main of which is the problem of illumination of the tissue of interest and the possible mutations caused by the action of a femtosecond laser. ${ }^{42}$ With this regard, the nonlinear microscopy requires tissue biopsy, and in most cases, cannot be used in routine practice as a method of the assessment of radiation-induced damage of the internal organs extracellular matrix. On the contrary, OCT is a fairly simple minimally invasive procedure.

The CP OCT method allows not only to describe the microstructure of biological tissues, but also to assess the ability of their connective-tissue matrix to change the polarization features of a probing radiation. With regard to the rectum and bladder, this is possible due to random cross scattering. CP OCT provides information about the damage of the microstructure of depolarizing tissue components, among which collagen fibers predominate. With the help of this method, it was shown that inflammation and invasive cancer significantly alter the ability of the tissue to cross-scattering. ${ }^{34,43-46}$

In our study, the CP OCT method was first used to study the features of the connective-tissue matrix of internal organs at various times after exposure of increasing doses of gamma radiation. The study showed a reduction in cross-scattering properties of the bladder and rectum tissue in 1 day and 1 week after exposure in doses of 10 and $40 \mathrm{~Gy}$ with a subsequent increase of IDF 1 month after exposure to a dose of $10 \mathrm{~Gy}$ and pronounced lowering of IDF after exposure to a dose of $40 \mathrm{~Gy}$. Reduction of this indicator in the early times after irradiation can reflect processes of collagen degradation, the subsequent IDF increase- - the restoration of the extracellular matrix. The pronounced IDF decrease after irradiation in a dose of 40 Gy can indicate a persistent disorganization of collagen, which is associated with exposure to high doses of radiation.

Quantitative analysis of the features of the connective-tissue framework after radiation exposure provided additional information about the processes occurring in the collagen-containing structures of normal tissues under the influence of ionizing radiation. The high correlation between values of the SHG signal intensities and IDF of the bladder and rectum that was found will help to noninvasively assess the status of extracellular matrix after exposure to even low doses of radiation without performing a histological study. This makes it possible to use CP OCT in clinical practice to diagnose and monitor the treatment of radiation-induced changes in normal tissues.

\section{Disclosures}

The authors have no relevant financial interests in this article and no potential conflicts of interest to disclose.

\section{Acknowledgments}

This work was supported by the Russian Foundation for Basic Research (project \# 16-02-00670 and project \# 16-07-00655).

\section{References}

1. DCTC, NCI, NIH, and DNNS, "Common terminology criteria for adverse events, version 5.0," Cancer Therapy Evaluation Program (2017).

2. W. Dörr and J. H. Hendry, "Consequential late effects in normal tissues," Radiother. Oncol. 61(3), 223-231 (2001).

3. J. Yarnold and M. C. V. Brotons, "Pathogenetic mechanisms in radiation fibrosis," Radiother. Oncol. 97(1), 149-161 (2010).

4. C. Fiorino et al., "Predictive models of toxicity in external radiotherapy," Radiother. Oncol. 93, 153-167 (2009).

5. J. Jaal and W. Dorr, "Radiation effects on cellularity, proliferation and EGFR expression in mouse bladder urothelium," Radiat. Res. 173(4), 479-485 (2010).

6. B. Davoudi et al., "Optical coherence tomography platform for microvascular imaging and quantification: initial experience in late oral radiation toxicity patients," J. Biomed. Opt. 18(7), 076008 (2013).

7. A. V. Maslennikova et al., "Use of optical coherence tomography for prognosis of the severity of oral mucositis," Vopr. Onkol. 55(5), 572-579 (2009).

8. T. Yasui et al., "In vivo time-lapse imaging of skin burn wound healing using second-harmonic generation microscopy," Proc. SPIE 8948, 89480B (2014).

9. T. H. Tsai et al., "Visualizing laser-skin interaction in vivo by multiphoton microscopy," J. Biomed. Opt. 14(2), 024034 (2009).

10. W. Denk, J. H. Strickler, and W. W. Webb, "Two-photon laser scanning fluorescence microscopy," Science 248(4951), 73-76 (1990).

11. T. A. Theodossiou et al., "Second harmonic generation confocal microscopy of collagen type I from rat tendon cryosections," Biophys. J. 91(12), 4665-4677 (2006).

12. S. Seidenari et al., "Multiphoton laser microscopy and fluorescence lifetime imaging for the evaluation of the skin," Dermatol. Res. Pract. 2012, 1-8 (2012). 
13. R. M. Williams, W. R. Zipfel, and W. W. Webb, "Interpreting secondharmonic generation images of collagen I fibrils," Biophys. J. 88(2), 1377-1386 (2005).

14. C. B. Raub et al., "Image correlation spectroscopy of multiphoton images correlates with collagen mechanical properties," Biophys. J. 94(6), 2361-2373 (2008).

15. T. H. Tsai et al., "Multiphoton microscopy in dermatological imaging," J. Dermatol. Sci. 56(1), 1-8 (2009).

16. A. S. Huang et al., "Sources of structural autofluorescence in the human trabecular meshwork," Invest. Ophthalmol. Visual Sci. 54(7), 48134820 (2013).

17. S. R. Kantelhardt et al., "In vivo multiphoton tomography and fluorescence lifetime imaging of human brain tumor tissue," J. Neurooncol. 127(3), 473-482 (2016).

18. W. S. Chen et al., "Multiphoton microscopic imaging of human normal and cancerous oesophagus tissue," J. Microsc. 253(1), 79-82 (2014).

19. R. Cicchi et al., "Non-linear imaging and characterization of atherosclerotic arterial tissue using combined two photon fluorescence, second-harmonic generation and CARS microscopy," Proc. SPIE 8948, 894807 (2014).

20. E. Gubarkova et al., "Characterization of atherosclerotic plaques by cross-polarization optical coherence tomography," Proc. SPIE 9689, 96893F (2016).

21. K. Tilbury et al., "Second harmonic generation microscopy analysis of extracellular matrix changes in human idiopathic pulmonary fibrosis," J. Biomed. Opt. 19(8), 086014 (2014).

22. L. S. Dolin et al., "OCT fundamentals and clinical applications of endoscopic OCT," in Handbook of Coherent-Domain Optical Methods: Biomedical Diagnostics, Environmental Monitoring and Material Science, V. Tuchin, Eds., 2nd ed., Vol. 2, pp. 999-1064, SpringerVerlag, New York (2013).

23. E. V. Zagaynova et al., "Optical coherence tomography monitoring of surgery in oncology," Handb. Biophotonics 2(24), 337-376 (2012).

24. E. B. Kiselyova et al., "Numerical analysis of cross-polar optic coherent tomography images in functional diagnostics of intestinal diseases by the condition of oral soft tissues," Sovrem. Tehnol. Med. 4, 32-39 (2011).

25. A. Maslennikova et al., "Monitoring of radiation cystitis development using direct and polarization-sensitive optical coherence tomography," Radiother. Oncol. 88(2), 243 (2008).

26. A. Maslennikova et al., "Effects of gamma irradiation on collagen damage and remodeling," Int. J. Radiat. Biol. 91(3), 240-247 (2015).

27. A. Alex et al., "Three-dimensional multiphoton/optical coherence tomography for diagnostic applications in dermatology," $J$. Biophotonics 6(4), 352-362 (2013).

28. N. Wang et al., "Monitoring skin trauma healing in mice using secondharmonic generation combined with optical coherence tomography," IEEE Photonics J. 9(4), 3700112 (2017).

29. P. Wilder-Smith et al., "Noninvasive imaging of oral premalignancy and malignancy," J. Biomed. Opt. 10(5), 051601 (2005).

30. S. Tang et al., "Combined multiphoton microscopy and optical coherence tomography using a 12 -fs broadband source," J. Biomed. Opt. 11(2), 020502 (2006).
31. E. Kiseleva et al., "Differential diagnosis of human bladder mucosa pathologies in vivo with cross-polarization optical coherence tomography," Biomed. Opt. Express 6(4), 1464-1476 (2015).

32. S. D. Giattina et al., "Assessment of coronary plaque collagen with polarization sensitive optical coherence tomography (PS-OCT)," Int. J. Cardiol. 107(3), 400-409 (2006).

33. M. J. Turunen et al., "Effects of tissue fixation and dehydration on tendon collagen nanostructure," Struct. Biol. 199(3), 209-215 (2017).

34. V. M. Gelikonov and G. V. Gelikonov, "New approach to cross-polarized optical coherence tomography based on orthogonal arbitrarily polarized modes," Laser Phys. Lett. 3(9), 445-451 (2006).

35. J. M. Schmitt and S. H. Xiang, "Cross-polarized backscatter in optical coherence tomography of biological tissue," Opt. Lett. 23(13), 10601062 (1998)

36. S. S. Kuznetsov et al., "Multiphoton microscopy in the study of morphological characteristics of radiation-induced injuries of the bladder," Sovrem. Tehnol. Med. 8(2), 31-39 (2016).

37. L. B. Mostaço-Guidolin et al., "Evaluation of texture parameters for the quantitative description of multimodal nonlinear optical images from atherosclerotic rabbit arteries," Phys. Med. Biol. 56(16), 5319-5334 (2011).

38. R. Cicchi et al., "Characterization of collagen and cholesterol deposition in atherosclerotic arterial tissue using non-linear microscopy," J. Biophotonics 7(1-2), 135-143 (2014).

39. C. Bayan et al., "Fully automated, quantitative, noninvasive assessment of collagen fiber content and organization in thick collagen gels," $J$. Appl. Phys. 105(10), 102042 (2009).

40. K. E. Frisch et al., "Quantification of collagen organization using fractal dimensions and Fourier transforms," Acta Histochem. 114(2), 140-144 (2012).

41. W. Hu et al., "Characterization of collagen fibers by means of texture analysis of second harmonic generation images using orientation dependent gray level co-occurrence matrix method," J. Biomed. Opt. 17(2), 026007 (2012).

42. J. M. D. Cruz et al., "Feasibility of using multiphoton excited tissue autofluorescence for in vivo human histopathology," Biomed. Opt. Express 1(5), 1320-1330 (2010).

43. R. Kuranov et al., "Complementary use of cross-polarization and standard OCT for differential diagnosis of pathological tissues," Opt. Express 10(15), 707-713 (2002).

44. N. Gladkova et al., "Cross-polarization optical coherence tomography for early bladder-cancer detection: statistical study," J. Biophotonics 4(7-8), 519-532 (2011).

45. E. V. Gubarkova et al., "Multi-modal optical imaging characterization of atherosclerotic plaques," J. Biophotonics 9(10), 1009-1020 (2016).

46. N. Gladkova et al., "Combined use of fluorescence cystoscopy and cross-polarization OCT for diagnosis of bladder cancer and correlation with immunohistochemical markers," J. Biophotonics 6(9), 687-698 (2013).

Biographies for the authors are not available. 